Published in "Journal of Antimicrobial Chemotherapy 74(7): 1934-1939, 2019"

which should be cited to refer to this work.

\title{
ESBLs and resistance to ceftazidime/avibactam and ceftolozane/tazobactam combinations in Escherichia coli and Pseudomonas aeruginosa
}

\author{
José-Manuel Ortiz de la Rosa ${ }^{1}$, Patrice Nordmann ${ }^{1-4}$ and Laurent Poirel ${ }^{1-3 *}$ \\ ${ }^{1}$ Emerging Antibiotic Resistance Unit, Medical and Molecular Microbiology, Department of Medicine, University of Fribourg, Fribourg, \\ Switzerland; ${ }^{2}$ Swiss National Reference Centre for Emerging Antibiotic Resistance, Fribourg, Switzerland; ${ }^{3}$ INSERM European Unit (LEA), \\ IAME, Paris, France; ${ }^{4}$ University of Lausanne and University Hospital Centre, Lausanne, Switzerland \\ ${ }^{*}$ Corresponding author. Medical and Molecular Microbiology Unit, Chemin du Musée 18, CH-1700 Fribourg, Switzerland. \\ Tel: +41 26300 9582; E-mail: laurent.poirel@unifr.ch
}

Received 11 January 2019; returned 14 February 2019; revised 27 February 2019; accepted 15 March 2019

\begin{abstract}
Objectives: To evaluate the efficacy of the recently launched $\beta$-lactam/ $\beta$-lactamase inhibitor combinations ceftazidime/avibactam and ceftolozane/tazobactam against ESBL-producing Escherichia coli and Pseudomonas aeruginosa strains.
\end{abstract}

\begin{abstract}
Methods: A series of ESBL-encoding genes (bla $a_{\mathrm{TEM}}, b a_{\mathrm{SHV}}$, bla $a_{\mathrm{CTX}-\mathrm{M}}$, bla $a_{\mathrm{VEB}}, b l a_{\mathrm{PER}}, b l a_{\mathrm{GES}}$ and $b l a_{\mathrm{BEL}}$ ) was cloned and expressed in E. coli or P. aeruginosa recipient strains. Cultures of E. coli TOP10 harbouring recombinant plasmids and therefore producing the different ESBLs tested were grown in order to perform measurements of catalytic activities, using benzylpenicillin, ceftazidime and ceftolozane as substrates. $\mathrm{IC}_{50} \mathrm{~s}$ were additionally determined for clavulanic acid, tazobactam and avibactam.
\end{abstract}

Results: We showed here an overall better activity of ceftazidime/avibactam compared with ceftolozane/tazobactam toward ESBL-producing E. coli and P. aeruginosa. Several ESBLs of the GES, PER and BEL types conferred resistance to ceftolozane/tazobactam in E. coli and P. aeruginosa. For GES-6 and PER-1 producers, resistance to ceftolozane/tazobactam could be explained by a high hydrolysis of ceftolozane and a low activity of tazobactam as an inhibitor. On the other hand, PER-producing P. aeruginosa also exhibited resistance to ceftazidime/avibactam.

Conclusions: Altogether, the results show that the ESBL PER-1, which is widespread worldwide, may be a source of resistance to both ceftolozane/tazobactam and ceftazidime/avibactam. Excellent activity of ceftazidime/avibactam was highlighted for both ESBL-producing E. coli and ESBL-producing P. aeruginosa.

\section{Introduction}

MDR including resistance to broad-spectrum cephalosporins and ultimately to carbapenems is nowadays commonly observed worldwide in Enterobacteriaceae and in Pseudomonas aeruginosa. ${ }^{1}$ Resistance to broad-spectrum cephalosporins may occur by different mechanisms, including permeability defects and efflux overproduction. However, production of broadspectrum $\beta$-lactamases is the most significant mechanism leading to such resistance. ${ }^{2}$ Hence, resistance to cephalosporins may occur through overproduction of AmpC $\beta$-lactamases, for which corresponding genes may be intrinsic in some species (such as P. aeruginosa, Enterobacter cloacae and Serratia marcescens) or acquired in Klebsiella pneumoniae or Escherichia coli, or by the acquisition of ESBLS.

Recently, two novel drug combinations have been launched, namely ceftazidime/avibactam and ceftolozane/tazobactam. ${ }^{3}$
Avibactam is a non- $\beta$-lactam $\beta$-lactamase inhibitor with activity against Ambler class A ESBLs and class C AmpCs, thus potentiating the activity of ceftazidime. ${ }^{4}$ Interestingly, it is active against KPCtype enzymes, which are weakly inhibited by clavulanic acid and tazobactam. ${ }^{4}$ However, some KPC variants have been identified, such as KPC-31 or KPC-35, that were shown to confer resistance to ceftazidime/avibactam in clinical K. pneumoniae isolates. ${ }^{5,6}$

Ceftolozane is a novel cephalosporin derivative of ceftazidime with intrinsic broad activity, which is furthermore not hydrolysed by most broad-spectrum $\beta$-lactamases, namely ESBLS and AmpCs. ${ }^{7}$ The ceftolozane/tazobactam association is particularly active against MDR (including carbapenem-resistant) P. aeruginosa. ${ }^{8}$

Although the most commonly identified ESBLs encountered in E. coli are CTX-M-type enzymes, along with TEM and SHV derivatives, those mainly encountered in P. aeruginosa are SHV-, VEB-, 
GES- and PER-like enzymes, and BEL-, TEM- and CTX-M-like enzymes have been rarely identified. ${ }^{9}$ Furthermore, the carbapenem-hydrolysing KPC enzymes possess an ESBL hydrolysis spectrum, but additionally hydrolyse carbapenems. They are increasingly identified in Enterobacteriaceae (mainly K. pneumoniae, but also $E$. coli), but remain rarely identified in $P$. aeruginosa. ${ }^{10}$ One specific group of $\beta$-lactamases quite frequently identified in $P$. aeruginosa corresponds to GES enzymes, with GES-1 being an ESBL-sparing carbapenem, ${ }^{11}$ whereas variants GES-2 and GES-5 possess significant carbapenemase activity. ${ }^{12-14}$ GES-6, also possessing significant carbapenemase activity and originally identified in a K. pneumoniae isolate from Greece, ${ }^{15}$ has recently been identified in P. aeruginosa. ${ }^{16}$ We recently showed that GES-6 production is the source of acquired resistance to ceftolozane/tazobactam while not affecting the susceptibility to ceftazidime/avibactam. ${ }^{17}$

The objective of our study was therefore to evaluate the relative impact of different ESBLs on susceptibility to ceftolozane/tazobactam and to ceftazidime/avibactam, either in E. coli or in P. aeruginosa backgrounds. Those two bacterial species were retained since $E$. coli represents a common community-acquired pathogen and $P$. aeruginosa a common hospital-acquired pathogen.

\section{Materials and methods}

\section{Bacterial isolates}

A series of ESBL-encoding genes was cloned and expressed in E. coli or P. aeruginosa recipient strains. Those ESBLs were derivatives of bla $a_{\mathrm{TEM}}$, bla $_{\mathrm{SHV}}$, bla $_{\mathrm{CTX}-\mathrm{M}}, \mathrm{bla} a_{\mathrm{VEB}}, b l a_{\mathrm{PER}}, b_{\mathrm{GES}}$ and bla $a_{\mathrm{BEL}}$. Donor strains are listed in Table 1. In addition, the two carbapenemase genes bla $a_{\mathrm{KPC}-2}$ and bla $a_{\mathrm{VIM}-2}$ were added. P. aeruginosa PAO1 and E. coli TOP10 were used as recipient strains. ${ }^{17}$

\section{Cloning experiments}

Cloning of all ESBL-encoding genes was performed in the shuttle and broad-host range pUCp24 using PCR amplicons encompassing the entire coding sequence of all respective genes. ${ }^{18}$ Primers used for PCR amplification of the ESBL genes are listed in Table S1 (available as Supplementary data at JAC Online). Electroporation of the recombinant plasmids and expression of the respective ESBL genes were performed in the E. coli TOP10 background. ${ }^{17}$ Subsequently, electroporation of those same recombinant plasmids was performed in the $P$. aeruginosa PAO1 background. Selection was made on plates containing $100 \mathrm{mg} / \mathrm{L}$ ticarcillin and $30 \mathrm{mg} / \mathrm{L}$ gentamicin for both $E$. coli and $P$. aeruginosa transformants.

\section{Susceptibility testing}

MIC values were determined in triplicate by Etest (AB bioMérieux; Solna, Sweden) or broth microdilution (for ceftolozane alone) as previously described. ${ }^{17}$ Interpretation was based on EUCAST breakpoints, ceftazidime/ avibactam resistance being defined as $>8 \mathrm{mg} / \mathrm{L}$ for Enterobacteriaceae and $P$. aeruginosa, and ceftolozane/tazobactam resistance as $>1 \mathrm{mg} / \mathrm{L}$ for Enterobacteriaceae and $>4 \mathrm{mg} / \mathrm{L}$ for $P$. aeruginosa. ${ }^{19}$ In order to further evaluate the respective contributions of the $\beta$-lactam agent (ceftazidime or ceftolozane) and that of the $\beta$-lactamase inhibitor (avibactam or tazobactam, respectively), MICs were determined not only at the usual ratio concentrations (inhibitor concentrations at $4 \mathrm{mg} / \mathrm{L}$ ), but also at higher concentrations ( 8 and $16 \mathrm{mg} / \mathrm{L}$, respectively).

\section{$\beta$-Lactamase activities}

Cultures of E. coli TOP10 harbouring recombinant plasmids and therefore producing the different ESBLs tested were grown overnight at $37^{\circ} \mathrm{C}$ in $1 \mathrm{~L}$ of brain heart infusion medium with amoxicillin (100 mg/L). The bacterial suspension was pelleted, resuspended in $10 \mathrm{~mL}$ of $100 \mathrm{mM}$ phosphate buffer $(\mathrm{pH} 7)$, disrupted by sonification ( $20 \mathrm{~min}$ for $30 \mathrm{~s}$ of sonication and $50 \mathrm{~s}$ of rest at $20 \mathrm{kHz}$ ) with a Vibra Cell 75186 (Thermo Fisher) and centrifuged for $1 \mathrm{~h}$ at $11000 \mathrm{~g}$ and $4^{\circ} \mathrm{C}$. $\beta$-Lactamase crude extracts were used for specific activity measurements performed at $30^{\circ} \mathrm{C}$ in $100 \mathrm{mM}$ sodium phosphate $(\mathrm{pH}$ 7.0). The initial rates of hydrolysis were determined with a GENESYS $10 \mathrm{~S}$ UV-Vis Spectrophotometer (Thermo Scientific). The following wavelengths/ absorption coefficients were used: benzylpenicillin, $232 \mathrm{~nm} /-1100$ $\mathrm{M}^{-1} \mathrm{~cm}^{-1}$; ceftazidime, $260 \mathrm{~nm} /-8660 \mathrm{M}^{-1} \mathrm{~cm}^{-1}$; and ceftolozane, $254 \mathrm{~nm} /-6810 \mathrm{M}^{-1} \mathrm{~cm}^{-1}$. IC $\mathrm{C}_{50} \mathrm{~s}$ were determined for clavulanic acid, tazobactam and avibactam. Various concentrations of these inhibitors were pre-incubated with the crude extract of the enzyme for $5 \mathrm{~min}$ at $30^{\circ} \mathrm{C}$ to determine the concentrations that reduced the hydrolysis rate of $100 \mu \mathrm{M}$ nitrocefin by $50 \%$. Results are expressed in micromolar units. The total protein content was measured using a Bradford assay.

\section{Results}

Among the 11 ESBL-producing P. aeruginosa clinical isolates, 10 were resistant to ceftolozane/tazobactam whereas most remained susceptible to ceftazidime/avibactam (Table 1). The ESBL-producing E. coli (CTX-M-15) was susceptible to both combinations. Notably, the KPC-2-producing $P$. aeruginosa isolate was susceptible to ceftazidime/avibactam, but resistant to ceftolozane/tazobactam.

Expression of the ESBL genes in E. coli TOP10 gave variable MIC values of ceftazidime/avibactam that remained in the susceptibility range (Table 2). However, the MIC of ceftazidime/avibactam for the bla $a_{\mathrm{PER}-1}$-positive recombinant strain was $8 \mathrm{mg} / \mathrm{L}$, which actually corresponds to the breakpoint value. This latter result was probably the consequence of a very high MIC of ceftazidime conferred by PER-1 activity, which was confirmed by the lowest MIC observed when combining ceftazidime with a higher concentration of avibactam (Table 2). MICs of ceftolozane/tazobactam for those recombinant $E$. coli strains were also variable, but, notably, several values remained in the resistance range, including those for the GES- 1 and GES- 6 producers, as well as for the PER-1, BEL- 1 and BEL-2 producers (Table 2). Notably, some high MIC values observed could be clearly explained by a combination of two features, namely a high hydrolysis rate toward ceftolozane and a lower inhibition by tazobactam, as for GES-6 and PER-1 (Table 3). Increasing the concentration of tazobactam significantly decreased the MIC of ceftazidime for the GES-1 and GES-5 producers, but not for the GES-6 and PER-1 producers (Table 2). The two carbapenemase-producing E. coli recombinant strains (KPC-2 and VIM-2) were resistant to ceftolozane/tazobactam, regardless of the tazobactam concentration used (Table 2).

Expression of the ESBL genes in P. aeruginosa PAO1 did not confer resistance to ceftazidime/avibactam except for the PER-1 producer (Table 4). This latter recombinant strain was the one with the highest MIC of ceftazidime, correlating with the poor activity of avibactam as an inhibitor toward PER-1 compared with other ESBLs (Table 3), this feature being confirmed by the still high MICs of ceftazidime observed even with higher concentrations of avibactam (Table 2). MICs of ceftolozane/tazobactam for recombinant $P$. aeruginosa strains varied considerably, with an overall high resistance rate observed for the GES-6, CTX-M-2, PER-1, BEL-1, BEL2 and SHV-2a producers (Table 4). Of note was the discrepancy observed between the GES-1 and GES-2 producers on the one 
Table 1. MICs for clinical isolates

\begin{tabular}{|c|c|c|c|c|c|c|c|c|c|c|c|c|}
\hline \multirow[b]{2}{*}{ Strain } & \multicolumn{12}{|c|}{ MIC (mg/L) } \\
\hline & IPM & MEM & CAZ & $C / A^{a}$ & $C / A^{b}$ & $C / A^{C}$ & $\mathrm{COZ}$ & $\mathrm{C} / \mathrm{T}^{\mathrm{d}}$ & $\mathrm{C} / \mathrm{T}^{\mathrm{e}}$ & $C / T^{f}$ & PIP & TZPd \\
\hline P. aeruginosa (GES-1) R1189 & 1 & 2 & 16 & 2 & 2 & 2 & 64 & 32 & 32 & 16 & 128 & 16 \\
\hline P. aeruginosa (GES-2) R184 & 16 & 32 & 64 & 4 & 2 & 2 & 32 & 16 & 8 & 4 & 256 & 128 \\
\hline P. aeruginosa (GES-5) R186 & 32 & 128 & 64 & 16 & 8 & 8 & 16 & 8 & 8 & 8 & 256 & 128 \\
\hline P. aeruginosa (GES-6) R3451 & 16 & 64 & 32 & 2 & 2 & 2 & 32 & 32 & 32 & 32 & 512 & 64 \\
\hline P. aeruginosa (CTX-M-2) R1188 & 1 & 8 & 32 & 8 & 4 & 4 & 16 & 4 & 1 & 1 & 256 & 32 \\
\hline E. coli (CTX-M-15) R1818 & 0.12 & $<0.06$ & 128 & 0.5 & 0.03 & 0.03 & 256 & 1 & 1 & 0.5 & $>512$ & 32 \\
\hline P. aeruginosa (PER-1) R1192 & 0.5 & 1 & $>512$ & 64 & 16 & 16 & 512 & 512 & 128 & 64 & 256 & 64 \\
\hline P. aeruginosa (BEL-1) R1185 & 1 & 2 & 32 & 4 & 2 & 2 & 16 & 8 & 8 & 8 & 128 & 64 \\
\hline P. aeruginosa (BEL-2) R1187 & 0.5 & 2 & 128 & 8 & 4 & 2 & 64 & 32 & 32 & 32 & 128 & 128 \\
\hline P. aeruginosa (VEB-1) R1205 & 8 & 16 & 256 & 8 & 4 & 4 & 64 & 64 & 32 & 32 & 128 & 32 \\
\hline P. aeruginosa (TEM-4) R1217 & 1 & 0.5 & 8 & 2 & 1 & 1 & 4 & 0.5 & 0.5 & 0.5 & 32 & 4 \\
\hline P. aeruginosa (SHV-2a) R136 & 1 & 2 & 32 & 4 & 4 & 2 & 8 & 4 & 4 & 2 & 512 & 256 \\
\hline P. aeruginosa (KPC-2) R96 & $>128$ & $>128$ & 256 & 8 & 4 & 2 & 64 & 64 & 32 & 32 & $>512$ & 512 \\
\hline P. aeruginosa (VIM-2) R166 & 128 & 64 & 64 & 64 & 64 & 64 & 256 & 256 & 256 & 256 & 256 & 32 \\
\hline
\end{tabular}

IPM, imipenem; MEM, meropenem; CAZ, ceftazidime; C/A, ceftazidime/avibactam; COZ, ceftolozane; C/T, ceftolozane/tazobactam; PIP, piperacillin; TZP, piperacillin/tazobactam.

MIC values indicated in bold for $\mathrm{C} / \mathrm{A}$ and $\mathrm{C} / \mathrm{T}$ are those corresponding to a categorization of resistance.

${ }^{\mathrm{a}}$ Avibactam at $4 \mathrm{mg} / \mathrm{L}$.

${ }^{\mathrm{b}} \mathrm{Avibactam}$ at $8 \mathrm{mg} / \mathrm{L}$.

${ }^{\mathrm{C} A v i b a c t a m}$ at $16 \mathrm{mg} / \mathrm{L}$.

${ }^{\mathrm{d}}$ Tazobactam at $4 \mathrm{mg} / \mathrm{L}$.

${ }^{\mathrm{e}}$ Tazobactam at $8 \mathrm{mg} / \mathrm{L}$.

- 'Tazobactam at $16 \mathrm{mg} / \mathrm{L}$.

hand and the GES-5 and GES-6 producers on the other hand. This result is in accordance with recent results that we have obtained showing that a serine residue at position 170 in either $\beta$-lactamase GES-5 or GES-6 (while GES-1 and GES-2, respectively, possess glycine and asparagine residues at that position) is responsible for a decreased sensitivity to tazobactam. Increasing the tazobactam concentration did not significantly modify the MICs of ceftolozane, except for the GES-1 recombinant strain (Table 2). As expected, the two carbapenemase-producing $P$. aeruginosa recombinant strains (KPC-2 and VIM-2) were also resistant to ceftolozane/ tazobactam.

Overall, two of the ESBL-producing P. aeruginosa recombinant strains were resistant to both ceftazidime/avibactam and ceftolozane/tazobactam combinations, namely those producing CTX-M-2 and PER-1.

\section{Discussion}

We showed here that ESBL acquisition may significantly counteract the activity of recently launched cephalosporin/ $\beta$-lactamase inhibitor combinations. Our data showed that ceftazidime/avibactam seems to be a very effective option, not only against ESBLproducing $E$. coli as reported in other studies, but also against ESBL-producing $P$. aeruginosa. In a recent study performed by Livermore et al., ${ }^{20}$ the prevalence of ESBL-producing (unknown determinant) Enterobacteriaceae susceptible to ceftazidime/avibactam was $99.7 \%$, among which only $3.8 \%$ initially remained susceptible to ceftazidime, thus showing an extremely high efficacy of the inhibitor. Looking at the susceptibility to ceftolozane/tazobactam among ESBL-producing $E$. coli recovered from healthcare infections in Latin America, Pfaller et al. ${ }^{21}$ found a rate of $9.2 \%$ of resistance, whereas it was very high (61.8\%) in ESBLproducing K. pneumoniae. Here we showed that production of ESBLs in E. coli does not impact the susceptibility to ceftolozane/ tazobactam, which is mainly due to the excellent activity of ceftolozane as an antibiotic.

In that same study from Pfaller et al., ${ }^{21}$ the rate of resistance to ceftolozane/tazobactam among ceftazidime-resistant $P$. aeruginosa was found to be $34.8 \%$, for which the mechanism of resistance to ceftazidime was not determined (probably corresponding to a majority of AmpC overproducers). In a recent surveillance programme among isolates from US hospitals and assessing the susceptibility of Enterobacteriaceae to ceftolozane/tazobactam, a rate of $87.5 \%$ of susceptibility was found among noncarbapenemase- and ESBL-producing isolates. Here we showed that production of an ESBL in $P$. aeruginosa may significantly affect the efficacy of ceftolozane/tazobactam, although susceptibility to ceftazidime/avibactam was preserved.

Of note, the very interesting activity of avibactam as a $\beta$-lactamase inhibitor was evidenced here with ceftazidime as substrate, but may also be extremely valuable by enhancing the $\beta$-lactam activity of various $\beta$-lactams. By increasing the concentrations of the respective inhibitors in the ceftazidime/avibactam and ceftolozane/tazobactam combinations, we observed some significant drops in terms of MIC values for only some recombinant strains, namely both the GES-1- and GES-5-producing E. coli and 
Table 2. MICs for E. coli recombinant isolates

\begin{tabular}{|c|c|c|c|c|c|c|c|c|c|c|c|c|}
\hline \multirow[b]{2}{*}{ Strain } & \multicolumn{12}{|c|}{ MIC (mg/L) } \\
\hline & IPM & MEM & CAZ & $C / A^{a}$ & $C / A^{b}$ & $C / A^{c}$ & $\mathrm{COZ}$ & $\mathrm{C} / \mathrm{T}^{\mathrm{d}}$ & $\mathrm{C} / \mathrm{T}^{\mathrm{e}}$ & $C / T^{f}$ & PIP & TZP \\
\hline E. coli TOP10 & 0.03 & 0.03 & 0.125 & 0.25 & 0.25 & 0.12 & 0.5 & 0.125 & 0.125 & 0.125 & 2 & 0.5 \\
\hline E. coli TOP10 + GES-1 & 0.12 & 0.03 & 8 & 0.25 & 0.25 & 0.12 & 32 & 8 & 0.5 & 0.25 & 16 & 1 \\
\hline E. coli TOP10 + GES-2 & 0.25 & 0.03 & 4 & 0.25 & 0.25 & 0.12 & 2 & 0.25 & 0.25 & 0.25 & 8 & 2 \\
\hline E. coli TOP10 + GES-5 & 1 & 0.5 & 8 & 0.5 & 0.25 & 0.12 & 8 & 8 & 0.25 & 0.25 & 32 & 16 \\
\hline E. coli TOP10 + GES-6 & 0.25 & 0.12 & 64 & 1 & 1 & 1 & 128 & 64 & 32 & 16 & 256 & 32 \\
\hline E. coli TOP10 + CTX-M-2 & 0.25 & 0.03 & 8 & 0.5 & 0.25 & 0.12 & 2 & 0.5 & 0.5 & 0.25 & 16 & 2 \\
\hline E. coli TOP10 + CTX-M-15 & 0.25 & 0.03 & 4 & 0.25 & 0.25 & 0.12 & 4 & 0.25 & 0.25 & 0.25 & 4 & 2 \\
\hline E. coli TOP10 + PER-1 & 0.5 & 0.03 & 512 & 8 & 0.25 & 0.25 & 512 & 128 & 128 & 64 & 8 & 4 \\
\hline E. coli TOP10 + BEL-1 & 0.25 & 0.03 & 16 & 0.5 & 0.25 & 0.12 & 8 & 4 & 2 & 1 & 8 & 8 \\
\hline E. coli TOP10 + BEL-2 & 0.25 & 0.03 & 32 & 0.5 & 0.25 & 0.12 & 16 & 8 & 2 & 2 & 16 & 2 \\
\hline E. coli TOP10 + VEB-1 & 0.25 & 0.03 & 16 & 0.5 & 0.25 & 0.12 & 0.5 & 0.5 & 0.25 & 0.25 & 128 & 16 \\
\hline E. coli TOP10 + TEM-4 & 0.25 & 0.03 & 2 & 0.25 & 0.25 & 0.12 & 0.5 & 0.25 & 0.25 & 0.25 & 8 & 2 \\
\hline E. coli TOP10 + SHV-2a & 0.25 & 0.03 & 8 & 0.25 & 0.25 & 0.12 & 4 & 1 & 0.25 & 0.25 & 16 & 4 \\
\hline E. coli TOP10 + KPC-2 & 8 & 2 & 16 & 0.5 & 0.25 & 0.12 & 8 & 8 & 8 & 8 & 64 & 32 \\
\hline E. coli TOP10 + VIM-2 & 8 & 4 & 64 & 32 & 16 & 16 & 512 & 128 & 128 & 128 & 32 & 32 \\
\hline
\end{tabular}

- IPM, imipenem; MEM, meropenem; CAZ, ceftazidime; C/A, ceftazidime/avibactam; COZ, ceftolozane; C/T, ceftolozane/tazobactam; PIP, piperacillin; TZP, piperacillin/tazobactam.

MIC values indicated in bold for $\mathrm{C} / \mathrm{A}$ and $\mathrm{C} / \mathrm{T}$ are those corresponding to a categorization of resistance.

a Avibactam at $4 \mathrm{mg} / \mathrm{L}$.

(1) ${ }^{\mathrm{b}}$ Avibactam at $8 \mathrm{mg} / \mathrm{L}$.

'Avibactam at $16 \mathrm{mg} / \mathrm{L}$.

${ }^{\mathrm{d}}$ Tazobactam at $4 \mathrm{mg} / \mathrm{L}$.

- ${ }^{\mathrm{e}}$ Tazobactam at $8 \mathrm{mg} / \mathrm{L}$.

( ${ }^{\mathrm{f}}$ Tazobactam at $16 \mathrm{mg} / \mathrm{L}$.

Table 3. Specific $\beta$-lactamase activities and $\mathrm{IC}_{50} \mathrm{~S}$ for $\beta$-lactamase inhibitors toward ESBLS

\begin{tabular}{|c|c|c|c|c|c|c|}
\hline \multirow[b]{2}{*}{ Enzyme } & \multicolumn{3}{|c|}{ Specific activity ( $\mu \mathrm{mol} \min ^{-1} \mathrm{mg}^{-1}$ ) } & \multicolumn{3}{|c|}{$\mathrm{IC}_{50}(\mu \mathrm{M})$} \\
\hline & benzylpenicillin & ceftazidime & ceftolozane & avibactam & tazobactam & clavulanic acid \\
\hline GES-1 & 2.8 & 0.4 & 0.4 & 1.9 & 0.7 & 3.5 \\
\hline GES-2 & 2.4 & 0.3 & 0.2 & 0.8 & 0.3 & 1.5 \\
\hline GES-6 & 3.2 & 0.3 & 0.7 & 3.7 & 7.3 & 78 \\
\hline GES-5 & 1.9 & 0.3 & 0.1 & 3.2 & 9 & 80 \\
\hline CTX-M-2 & 12 & 0.2 & 0.06 & 0.1 & 0.1 & 0.06 \\
\hline CTX-M-15 & 2 & 0.3 & 0.2 & 0.5 & 0.3 & 0.7 \\
\hline PER-1 & 7 & 0.6 & 2.2 & 10.4 & 4.2 & 4.7 \\
\hline BEL-1 & 10 & 0.3 & 0.1 & 0.1 & 2.6 & 0.4 \\
\hline BEL-2 & 2.5 & 0.4 & 0.3 & 0.35 & 0.4 & 0.4 \\
\hline VEB-1 & 17 & 0.2 & 0.07 & 0.01 & 0.05 & 0.3 \\
\hline TEM-4 & 5 & 0.2 & 0.09 & 0.5 & 0.01 & 0.1 \\
\hline SHV-2a & 15 & 0.3 & 0.2 & 0.01 & 0.04 & 0.05 \\
\hline KPC-2 & 12 & 0.3 & 0.3 & 3.9 & 50 & 130 \\
\hline VIM-2 & 6 & 0.4 & 1 & $>500$ & $>500$ & $>500$ \\
\hline
\end{tabular}

$P$. aeruginosa strains, respectively, while they remained almost unchanged for all the others, including the GES-6 producers. This highlights further that high MICs of the two drug combinations conferred by GES- 1 and GES- 5 might be counteracted by increased concentrations of the inhibitor. However, pharmacokinetic/pharmacodynamic data measured for those drug combinations show that the respective $\beta$-lactam/ $\beta$-lactamase inhibitor ratio remains stable in urine. ${ }^{22,23}$ 
Table 4. MICs for $P$. aeruginosa recombinant isolates

\begin{tabular}{|c|c|c|c|c|c|c|c|c|c|c|c|c|}
\hline \multirow[b]{2}{*}{ Strain } & \multicolumn{12}{|c|}{ MIC (mg/L) } \\
\hline & IPM & MEM & CAZ & $C / A^{a}$ & $C / A^{b}$ & $C / A^{c}$ & $\mathrm{COZ}$ & $\mathrm{C} / \mathrm{T}^{\mathrm{d}}$ & $\mathrm{C} / \mathrm{T}^{\mathrm{e}}$ & $C / T^{f}$ & PIP & TZPd \\
\hline PA01 & 0.5 & 0.5 & 1 & 1 & 1 & 1 & 2 & 0.5 & 0.5 & 0.5 & 4 & 1 \\
\hline PAO1+GES-1 & 1 & 0.5 & 64 & 1 & 1 & 1 & 32 & 16 & 8 & 2 & 64 & 4 \\
\hline PAO1+GES-2 & 1 & 1 & 16 & 2 & 2 & 2 & 8 & 2 & 0.5 & 0.5 & 16 & 8 \\
\hline PAO1+GES-5 & 4 & 16 & 32 & 2 & 2 & 2 & 8 & 16 & 2 & 2 & 32 & 16 \\
\hline PAO1+GES-6 & 4 & 8 & 64 & 1 & 1 & 1 & 64 & 32 & 32 & 32 & 128 & 32 \\
\hline PAO1+CTX-M-2 & 1 & 0.5 & 128 & 8 & 4 & 2 & 32 & 8 & 8 & 8 & 512 & 256 \\
\hline $\mathrm{PAO} 1+\mathrm{CTX}-\mathrm{M}-15$ & 1 & 0.5 & 8 & 2 & 2 & 2 & 4 & 1 & 1 & 0.5 & 16 & $8 / 4$ \\
\hline PAO1 + PER-1 & 1 & 1 & $>512$ & 64 & 32 & 16 & $>512$ & $>512$ & 256 & 128 & 128 & 64 \\
\hline PAO1+BEL-1 & 1 & 0.5 & 16 & 2 & 2 & 2 & 16 & 8 & 8 & 8 & 128 & 64 \\
\hline PAO1+BEL-2 & 1 & 0.5 & 128 & 4 & 4 & 2 & 64 & 16 & 16 & 16 & 64 & 32 \\
\hline PAO1+VEB-1 & 1 & 0.5 & 2 & 1 & 1 & 1 & 0.5 & 0.5 & 0.25 & 0.25 & 128 & 16 \\
\hline PAO1+TEM-4 & 1 & 1 & 4 & 2 & 1 & 1 & 4 & 0.5 & 0.25 & 0.25 & 16 & 4 \\
\hline $\mathrm{PAO} 1+\mathrm{SHV}-2 \mathrm{a}$ & 1 & 1 & 64 & 4 & 2 & 2 & 16 & 8 & 8 & 8 & 256 & 128 \\
\hline $\mathrm{PAO} 1+\mathrm{KPC}-2$ & 32 & 128 & 256 & 4 & 2 & 2 & 64 & 64 & 64 & 64 & 256 & 256 \\
\hline PAO1 + VIM-2 & 32 & 32 & 32 & 32 & 32 & 32 & 512 & 256 & 256 & 256 & 64 & 64 \\
\hline
\end{tabular}

- IPM, imipenem; MEM, meropenem; CAZ, ceftazidime; C/A, ceftazidime/avibactam; COZ, ceftolozane; C/T, ceftolozane/tazobactam; PIP, piperacillin; TZP, piperacillin/tazobactam.

MIC values indicated in bold for C/A and C/T are those corresponding to a categorization of resistance.

${ }^{a}$ Avibactam at $4 \mathrm{mg} / \mathrm{L}$.

${ }^{\mathrm{b}}$ Avibactam at $8 \mathrm{mg} / \mathrm{L}$.

'Avibactam at $16 \mathrm{mg} / \mathrm{L}$.

${ }^{\mathrm{d}}$ Tazobactam at $4 \mathrm{mg} / \mathrm{L}$.

- ${ }^{\mathrm{e}}$ Tazobactam at $8 \mathrm{mg} / \mathrm{L}$.

( $\mathrm{f}$ Tazobactam at $16 \mathrm{mg} / \mathrm{L}$.

Further work is now needed to evaluate the respective activities of ceftazidime/avibactam and ceftolozane/tazobactam toward a large collection of ESBL-producing $E$. coli and $P$. aeruginosa clinical isolates.

\section{Funding}

This work was funded by the University of Fribourg, by the Swiss National Science Foundation (projects FNS-31003A_163432 and PNR7240AR40_173686) and by the INSERM, Paris, France.

\section{Transparency declarations}

None to declare.

\section{Supplementary data}

Table S1 is available as Supplementary data at JAC Online.

\section{References}

1 Potron A, Poirel L, Nordmann P. Emerging broad-spectrum resistance in Pseudomonas aeruginosa and Acinetobacter baumannii: mechanisms and epidemiology. Int J Antimicrob Agents 2015; 45: 568-85.

2 Bush K. Past and present perspectives on $\beta$-lactamases. Antimicrob Agents Chemother 2018; 62: e01076-18.
3 Wright H, Bonomo RA, Paterson DL. New agents for the treatment of infections with Gram-negative bacteria: restoring the miracle or false dawn? Clin Microbiol Infect 2017; 23: 704-12.

4 Sharma R, Park TE, Moy S. Ceftazidime-avibactam: a novel cephalosporin/ $\beta$-lactamase inhibitor combination for the treatment of resistant Gramnegative organisms. Clin Ther 2016; 38: 431-44.

5 Hemarajata P, Humphries RM. Ceftazidime/avibactam resistance associated with L169P mutation in the omega loop of KPC-2. J Antimicrob Chemother 2019; 74: 1241-3.

6 Barnes MD, Winkler ML, Taracila MA et al. Klebsiella pneumoniae carbapenemase-2 (KPC-2), substitutions at Ambler position Asp179, and resistance to ceftazidime-avibactam: unique antibiotic-resistant phenotypes emerge from $\beta$-lactamase protein engineering. MBio 2017; 8: e00528-17.

7 van Duin D, Bonomo RA. Ceftazidime/avibactam and ceftolozane/tazobactam: second-generation $\beta$-lactam/ $\beta$-lactamase inhibitor combinations. Clin Infect Dis 2016; 63: 234-41.

8 Giacobbe DR, Bassetti M, De Rosa FG et al. Ceftolozane/tazobactam: place in therapy. Expert Rev Anti Infect Ther 2018; 16: 307-20.

9 Naas T, Poirel L, Nordmann P. Minor extended-spectrum $\beta$-lactamases. Clin Microbiol Infect 2008; 14 Suppl 1: 42-52.

10 Munoz-Price LS, Poirel L, Bonomo RA et al. Clinical epidemiology of the global expansion of Klebsiella pneumoniae carbapenemases. Lancet Infect Dis 2013; 13: 785-96.

11 Poirel L, Le Thomas I, Naas T et al. Biochemical sequence analyses of GES-1, a novel class $A$ extended-spectrum $\beta$-lactamase, and the class 1 integron In52 from Klebsiella pneumoniae. Antimicrob Agents Chemother 2000; 44: 622-32. 
12 Poirel L, Weldhagen GF, Naas T et al. GES-2, a class A $\beta$-lactamase from Pseudomonas aeruginosa with increased hydrolysis of imipenem. Antimicrob Agents Chemother 2001; 45: 2598-603.

13 Poirel L, Carrër A, Pitout JD et al. Integron mobilization unit as a source of mobility of antibiotic resistance genes. Antimicrob Agents Chemother 2009; 53: 2492-8.

14 Bontron S, Poirel L, Nordmann P. In vitro prediction of the evolution of GES-1 $\beta$-lactamase hydrolytic activity. Antimicrob Agents Chemother 2015; 59: $1664-70$.

15 Vourli S, Giakkoupi P, Miriagou V et al. Novel GES/IBC extended-spectrum $\beta$-lactamase variants with carbapenemase activity in clinical enterobacteria. FEMS Microbiol Lett 2004; 234: 209-13.

16 Botelho J, Grosso F, Peixe L. Unravelling the genome of a Pseudomonas aeruginosa isolate belonging to the high-risk clone ST235 reveals an integrative conjugative element housing a bla $a_{\mathrm{GES}-6}$ carbapenemase. J Antimicrob Chemother 2018; 73: 77-83.

17 Poirel L, Ortiz De La Rosa JM, Kieffer $\mathrm{N}$ et al. Acquisition of extendedspectrum $\beta$-lactamase GES-6 leading to resistance to ceftolozanetazobactam combination in Pseudomonas aeruginosa. Antimicrob Agents Chemother 2018; 63: e01809-18.

18 West SE, Schweizer HP, Dall C et al. Construction of improved Escherichia( Pseudomonas shuttle vectors derived from pUC18/19 and sequence of the region required for their replication in Pseudomonas aeruginosa. Gene 1994; 148: 81-6.

19 EUCAST. Breakpoint Tables for Interpretation of MICs and Zone Diameters, Version 8.1, 2018. http://www.eucast.org/fil eadmin/src/media/PDFs/EUCAST_files/Breakpoint_tables/v_8.1_Breakpoint_ Tables.pdf.

20 Livermore DM, Meunier D, Hopkins KL et al. Activity of ceftazidime/avibactam against problem Enterobacteriaceae and Pseudomonas aeruginosa in the UK, 2015-16. J Antimicrob Chemother 2018; 73: 648-57.

21 Pfaller MA, Shortridge D, Sader HS et al. Ceftolozane-tazobactam activity against drug-resistant Enterobacteriaceae and Pseudomonas aeruginosa causing healthcare-associated infections in Latin America: report from an antimicrobial surveillance program (2013-2015). Braz J Infect Dis 2017; 21: 627-37.

22 Wooley M, Miller B, Krishna $G$ et al. Impact of renal function on the pharmacokinetics and safety of ceftolozane-tazobactam. Antimicrob Agents Chemother 2014; 58: 2249-55.

23 Sy SKB, Zhuang L, Sy S et al. Clinical pharmacokinetics and pharmacodynamics of ceftazidime-avibactam combination: a model-informed strategy for its clinical development. Clin Pharmacokinet 2019; 58: 545-64. 